\title{
Review \\ Review on Tympanic Membrane and Auditory Canal Regeneration by Biomaterial Intervention
}

\author{
Deepak Ranglani ${ }^{\dagger}$, Shubham Agiwal ${ }^{\dagger}$, Namdev More ${ }^{\dagger}$, Rohit Parkale ${ }^{\dagger}$, Vaibhav Shitole ${ }^{\dagger}$, \\ Aishwarya Rajaram Hiray ${ }^{\dagger}$, Govinda Kapusetti*
}

Department of Medical Devices National Institute of Pharmaceutical Education and Research, Ahmedabad

\author{
ARTICLE INFO

\section{Article History} \\ Received 14 June 2020 \\ Keywords \\ Tympanic membrane \\ auditory canal \\ tissue engineering \\ biomaterials \\ cells \\ growth factors \\ stem cells
}

Accepted 16 December 2020

\begin{abstract}
The impairment in the Tympanic Membrane (TM) and the External Auditory Canal (EAC) results in otitis media, tympanosclerosis, and perforations. The resulting reverberation of these pathologies is the loss of hearing, which eventually requires surgical treatment. The gold standard for the surgical procedures, autologous temporalis fascia, is reported with limited success. In recent times the biomaterial substitutes for this very purpose have carried off tremendous interest. The current review explicitly focuses on the last 10 years reports describing TM and EAC regeneration by biomaterial intervention. This review also provides an intelligible insight into various fabrication techniques for grafting to mimic TM-collagen's radial and circular patterns. In the end, we have reached the terms by highlighting the future of the biomaterials in contrast to the current challenges in TM and EAC regeneration. Overall, the review will be a better source for developing futuristic biomaterials for the discussed problem.
\end{abstract}

(C) 2021 The Authors. Published by Atlantis Press B.V. This is an open access article distributed under the CC BY-NC 4.0 license (http://creativecommons.org/licenses/by-nc/4.0/).

\section{INTRODUCTION}

The Tympanic Membrane (TM) is a cone-shaped membrane that separates the outer ear from the middle ear. The TM is an integral part of the ear, contributes majorly to the activity of hearing. Functionally, the collects and transmits sound vibrations covering the middle ear [1]. Anatomically it is a delicate, semi-opaque yet diaphanous and thin membrane structure, mainly made up of pars flaccida and pars tensa. The outer and inner lining of the pars tensa consists of the keratinising and cuboidal epitheliums and are separated by lamina propria. The lamina propria encloses collagen fibers arranged radially on the outside and rounded on the inside, along with the parabolic fibers, makes the pars tensa stiffer and thin [1]. The epithelial layer present in the external ear is separated from the inner layer by a connective tissue layer made up of collagen, fibroblasts, and elastic fibers. In brief, the sound conduction mechanism of TM occurs when the acoustic waves hit TM, resonance occurs, which will cause the ossicles to vibrate. In addition, follow a series of events through the central auditory pathway to promote hearing [2].

Tympanic membrane is a delicate tissue that is easily injured and develops further into a medical condition characterized by membrane perforations (holes), which further leads to complete or partial hearing loss, namely deafness.

Tympanic membrane is a delicate tissue, that is easily injured and develops further into a medical condition characterized by

"Corresponding author. Email: govindphysics@gmail.com

Authors having equal contributions. membrane perforations (holes), which further leads to complete or partial hearing loss, i.e., deafness [3]. The major etiological factors are acoustic trauma (loud noise), otitis media, ear infections, insertion of some foreign materials in the ear like tympanostomy tubes, earbuds, physical trauma, and so on [4].

Untreated perforations may progress to hearing loss, tinnitus (ringing of ears), Cholesteatoma (noncancerous skin growth often developed as a cyst), otalgia, otorrhea, and severe calcifications [5-7]. In general, the healing of perforations is mediated by epithelial migration [8]; First, the hyperplastic squamous epithelium at the perforation edges exhibits excessive keratinization, leading to the appearance of the keratin-covered gap. The second step is the proliferation of the layered squamous epithelium to cover the gap again, and the third step is the reconstruction of the three-layer sandwich structure of the membrane [9]. Thus, the smaller perforations are healed spontaneously. At the same time, the healing of larger perforations is very much impossible [10]. Various surgical methods are used to treat perforations like Myringoplasty (procedure of closure of the perforation present in pars tensa of the TM) and Tympanoplasty (myringoplasty along with ossicular reconstruction). The gold standard is autologous temporalis fascia. But, surgical intervention has its drawbacks like invasive procedures, discomfort, graft rejection, limitation in graft availability, and the procedure's cost [11]. Several sophisticated techniques related to regenerative treatment to treat perforated TM have also been reported using various approaches like cartilage-perichondrium, temporalis fascia due to its healing and acoustic properties [12], vein grafts, sclera, fat, inlay cartilage [13], the acellular dermis [4], and various paper patches [14]. The regenerative approaches include tissue engineering, and it is 
the stream of science the utilizes combination of cells, engineering methods, and aspects along with materials such as suitable biochemical and physicochemical factors to improve or replace biological tissues using three essential elements, (1) cells like fibroblasts, (2) growth Factors as regulatory factors, (3) biomaterials for the synthesis of scaffolds. They all can be used alone or even in combination, depending on the case and materials used by providing a suitable microenvironment $[15,16]$.

The current review discusses the recent reports on advanced materials for the regeneration of the TM and AEC. The primary role of the AEC is the perceiving sound waves and allowing them to hit the TM. The AEC is divided into an external and inner canal. The inner Auditory Canal (AC) is crucial for balance, and the external canal is solely responsible for the collection or perception of sound waves. The major diseases like adhesive otitis media and Cholesteatoma result in damage to the AC and TM leading to hearing loss. Another AC disease, like aural atresia, refers to an absence of the ear canal, and microtia indicates a small, abnormally shaped auricle. Microtia and aural atresia tend to occur simultaneously. Thus advancement in materials and regenerative approach is used for the reconstruction of the AC. Various polymers, stem cell therapy, tissue-engineering, fiber deposited scaffolds, and ceramics $[17,18]$ are extensively used to regenerate the TM and AEC in recent times [19].

Besides scaffold fabrication, this review also focuses on the development of materials for the TM and AC regeneration. The biomaterials include polymers, for instance, natural origin; silk fibroin, and chitosan, which specifically results in water-soluble patch formation. The polymers of biological origin have also been discussed, namely collagen and gelatin, which provide higher biocompatibility, bioactivity, and cell proliferation. Synthetic polymers like Polycaprolactone (PCL), Polybutylene terephthalate (PBOT/ PBT), Polylactic Acid (PLA), Polydimethylsiloxane (PDMS), and Poly Lactic-co-glycolic Acid (PLGA) are also discussed at length. These polymers have been used alone or in combination to form polymer composites to build on mechanical and structural properties to mimic the TM and AC properties. Various growth factor loaded biomaterials have also been confabulated in this review, including epidermal growth factor, basic fibroblast growth factor, and platelet-derived growth factor (PDGF), which mainly have the role of up-regulating the cell proliferation by providing a copacetic microenvironment to the cells. A recent regenerative therapy approach using stem cells mainly of embryonic origin has also been discussed at length $[20,21]$.

\section{IDEAL CHARACTERISTICS OF SCAFFOLD}

The features and properties of scaffold material are called for successful tissue regeneration and quality of tissue production. The material properties of the scaffold act as a pivot to ascertain the cellular response and fate. Under ideal conditions, in vitro or in vivo, the carrier material should lay out the specified properties. The scaffold characteristics such as morphology, porosity, mechanical properties, biological properties, shape, and biodegradability are considered critical components of better tissue regeneration. The most crucial factor in the scaffold characteristics is the biocompatibility, biodegradability, and healing effect.
The fabrication technique counts importance in obtaining appropriate characteristics of scaffold [22]. The scaffold's porosity is an important parameter that will induce cell integration by permeability and cell adhesion. The three-dimensional scaffold is typically porous with interconnected pore networks to facilitate cell nutrition, oxygen diffusion, and waste removal [23]. Hence, improved pore interconnectivity and porosity will aid in rapid tissue regeneration. Characteristics like biocompatibility are indispensable in controlling and managing the immune sensitivity to host response. The shape of the scaffold is another important parameter to control the mimicking of the regenerated tissue with native tissue [24]. Polymer fiber scaffolds of exact shape and size could help in the regeneration of nerve cells in the damaged after injury [25]. Mechanical properties ensure the strength of the scaffold to support cell development and growth. The mechanical properties and stability of the scaffold are optimized with the bioactive composition polymer. Thus composition Poly(ethylene oxide terephthalate) (PEOT)/PBT block copolymer was calculating by Young's modulus through compression testing [26].

\section{BIOMATERIALS FOR TM REGENERATION}

The advancements in material science and material engineering encouraged the investigation of various biomaterials. Development of multiple tools and understanding the material interaction with the physiological environment also led to the exploration of new biomaterials, specifically polymers. Tissue engineering is said as a blend of biomedical and material sciences for the growth of various cells to form tissues.

\subsection{Biomaterials Driven Regeneration}

Polymers are the best-known materials for tissue engineering since they are easy to fabricate and tuneable for various properties like mechanical, physical, and biological [27]. Calcium alginate was conventionally employed in TM regeneration. The cytotoxicity, deprived mechanical properties, and poor clinical results restrict its use at a later time. The water-soluble chitosan was found with suitable biological, mechanical, and structural features for TM regeneration. The chitosan patch derived TM tissue was reported for higher collagen density, better healing rate, better mechanical properties, improved biocompatibility, and biodegradability, compared to the spontaneously healed tissue [28]. Kim et al. [29] developed a $3 \mathrm{D}$ porous chitosan patch for TM regeneration. The highly organized pore size of the 3D chitosan scaffold $(140-230 \mu \mathrm{m})$ was able to generate high-quality TM as compared to conventional scaffold [29]. Interestingly, the 3D chitosan scaffold was reported for the regeneration of thicker TM than the native tissue. The result was highly motivational for the use of polymeric biomaterials for the regeneration of TM and other tissues.

The Silk Fibroin (SF) and Acellular Collagen (ACG) type 1/3 were used separately for the regeneration of TM. Figure 1 shows the otoscopic images of rat and pig TM after the SF and ACG implantation. It was found that a developed scaffold can improve the adhesion and proliferation of keratinocytes [30]. The mechanical properties and vibroacoustic properties were evaluated as an essential parameter in pigs and rats [30-32]. The tensile strength of SF 


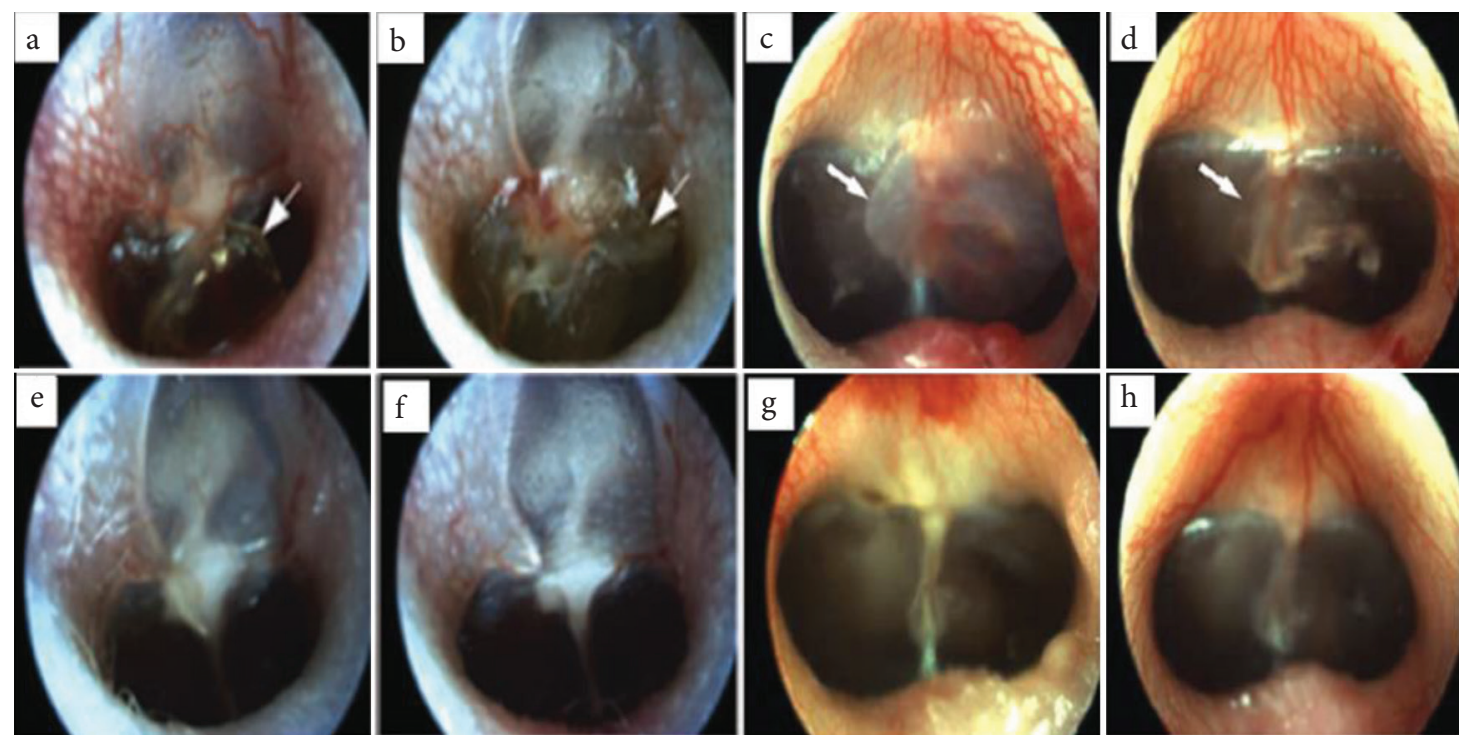

Figure 1 Otoscopic images of rat and pig's Tympanic Membrane (TM). (a-d) TM before grafting. (e) After using Silk Fibroin (SF) patch in rats. (f) After using Acellular Collagen (ACG) patch in rats. (g) After using SF patch in guinea pigs. (h) After using ACG patch in guinea pigs. The developed scaffolds had shown improved wound healing [31,32]

scaffold was reported to be higher $(42 \pm 2.2 \mathrm{MPa})$ than that of the paper patch $(34.4 \pm 1.7 \mathrm{MPa})$. On the other hand, the paper patch showed higher tensile strength than that of ACG scaffold (21.4 \pm 1.6 MPa). However, the elongation of ACG $(19.6 \pm 1.4 \mathrm{MPa})$ was higher than that of SF scaffold and paper patch [31]. This property of being flexible is indeed useful in vibroacoustic of TM. Hence, the team concluded that the composite of ACG and SF possessed the benefits of optimal mechanical strength, keratinocyte growth, improved structural veracity, early restoration of hearing, and accelerated wound healing, proving to be an alternative biomaterial for efficient TM regeneration [30-32].

Lee et al. [33] prepared a nanocomposite of SF and PCL and used electrospinning technology together with human Umbilical Cord Serum (UCS) for the scaffold. UCS is a mixture of many growth factors such as Epidermal Growth Factors (EGF), PDGF, acidic and basic Fibroblast Growth Factors (FGFs), hepatocyte growth factor, insulin-like growth factor-1, Transforming Growth Factor (TGF)- $\beta$, nerve growth factor, and substance $\mathrm{P}$, etc. [33]. They characterized the in vitro cellular events of the scaffolds (pure PCL, pure SF, PCL/SF, and PCL/SF/UCS) and found that the cell activity in PCL/SF/UCS was higher than the other scaffolds. The $\mathrm{PCL} / \mathrm{SF}$ and pure SF scaffolds are reported for outstanding development of F-actin by fluorescence imaging. At the same time, $\mathrm{PCL} / \mathrm{SF} / \mathrm{UCS}$ promoted the spread of cells on the entire surface, thus revealing cells' excellent viability and metabolic activity (Figure 2a). The SF and growth factors of UCS act as cell activation sites, promoting their attachment and proliferation in the PCL/SF/UCS scaffold. The acoustic capabilities of TM were also evaluated to ensure normal hearing in guinea pigs. After 21 days of implantation in PCL/SF/UCS, total healing was reported, while only $41 \%$ healing was claimed in the control group (paper patch). Auditory brainstem response threshold and single-point laser Doppler vibrometer results showed that compared with the control group, the vibration of the experimental group was significantly restored [33]. Therefore, in vitro cell viability studies and in vivo studies of subacute TM perforation provide an alternative biomaterial scaffold for TM regeneration, eliminating time-consuming and expensive surgical procedures. Recently, Moscato et al. [34] demonstrated the TM collagen type expression in dynamically cultured Human Mesenchymal Stromal Cells (hMSC) dynamically cultured on electrospun nonwoven constructs of star-branched PCL [34]. The research team conducted cell culture studies under static and dynamic conditions to understand collagen expression [34]. The dynamic culture was carried out under radial and circular strain on the scaffold, mimicking the natural TM collagen patterns. Cell viability by neutral red assay revealed the presence and distribution of viable hMSC in static, dynamically differentiated, and undifferentiated conditions, indicating a higher neutral red intensity in the dynamically cultured construct. Under dynamic and static conditions, in the differentiated and undifferentiated hMSCs on the PCL scaffold, the exciting gene expression results of collagen I, II, IV $\alpha-1$, and IV $\alpha-2$ were reported (Figure $2 b$ ). It was observed that under static conditions, differentiated constructs have higher expression of type I collagen than undifferentiated constructs In addition, the reduced expression in the dynamically differentiated constructs was discussed than in the statically differentiated constructs. In the static and dynamic differentiation groups, the expression of type II collagen was significantly higher than that of the undifferentiated control group. The results of collagen IV $\alpha-1$ expression are similar to the results of collagen I expression. Even by adjusting the culture conditions and distinguishing the medium, no statistical difference in the expression level of collagen IV $\alpha-2$ was observed [34]. IHC studies have shown that type II collagen is not significantly expressed under static differentiation and undifferentiated conditions, which confirms that proper differentiation conditions and the combination of TM bioreactors are necessary for the expression of type II collagen in hMSC. Therefore, this report is considered to be the standard for the differentiation of stromal cells into TM fibroblast lineage, which can be proved by means of hMSC, electrospinning scaffold, proper differentiation conditions and TM bioreactor to achieve effective TM regeneration. Taking into account the limitations of current autograft materials, Kozin et al. designed, produced, and evaluated 
a

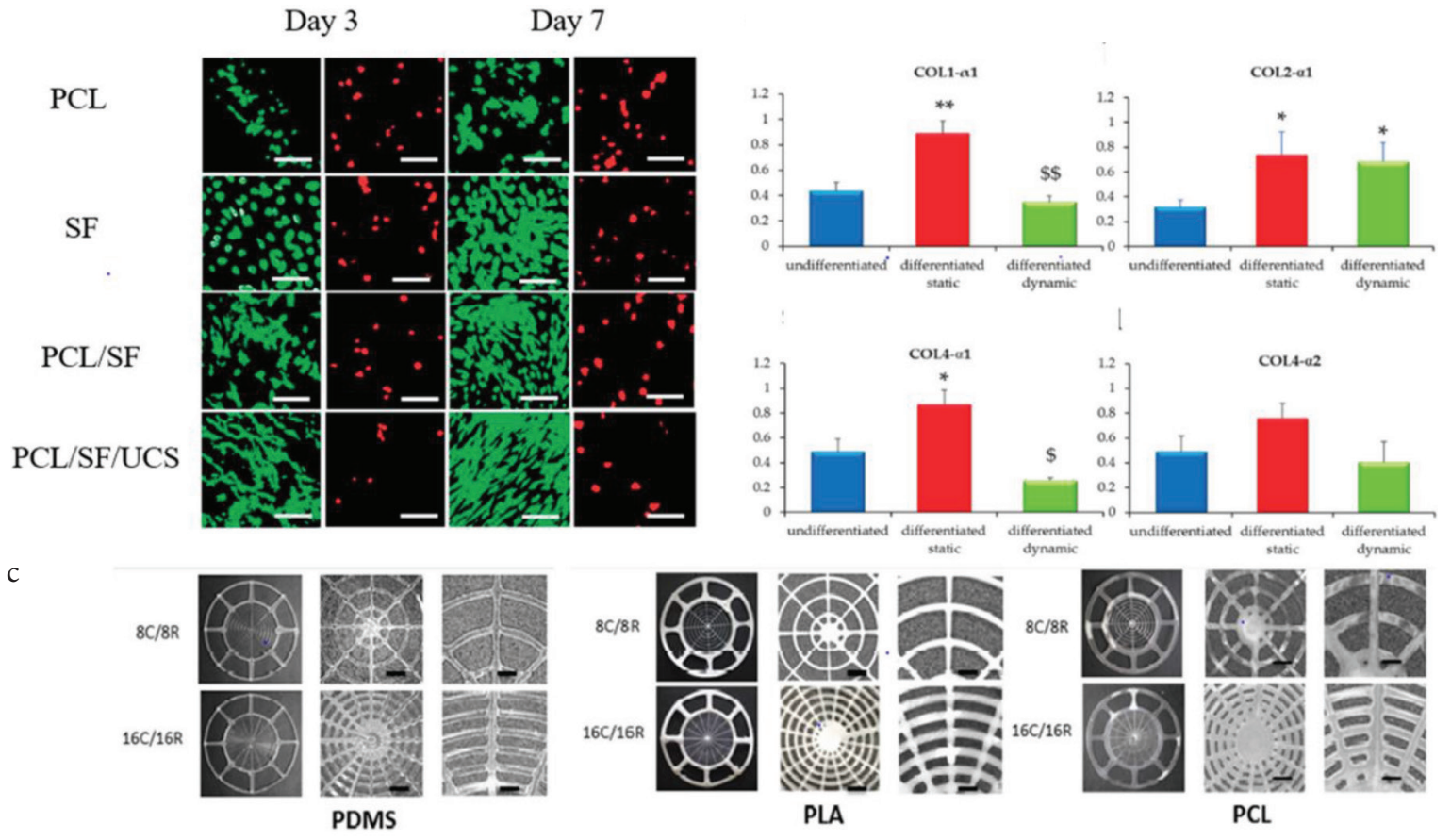

Figure 2 (a) Fluorescence image of the live (green) dead (red) assay of the pure Polycaprolactone (PCL), pure Silk Fibroin (SF), PCL/SF, PCL/SF/ Umbilical Cord Serum (UCS). (b) Collagen expression on hMSC's/*PCL for the expression of collagen I, collagen II, collagen IV $\alpha$ - 1 and collagen $\alpha-2$. (c) 3D printed image of the TM scaffolds prepared from Polydimethylsiloxane (PDMS), flex-Polylactic Acid (PLA), PCL biomaterials [33-35]. ${ }^{\star} p<0.05$, ${ }^{* *} p<0.01$ vs undifferentiated; ${ }^{s} p<0.05,{ }^{s \$} p<0.01$ vs differentiated statically cultured hMSC/ $/$ PCL constructs.

multi-material 3D printed TM graft scaffold. The team fabricated a scaffold with a blend of PDMS, flex-PLA, and PCL with an aspect ratio of $8 / 16$ in radial and circumferential filament arrangement (Figure 2c). They tried to imitate the Extracellular Matrix (ECM) of human TM by filling a biodegradable hydrogel (fibrin collagen) in the 3D printed scaffold skeleton. The digital opto-electronic holography study shows that all TM grafts exhibit a simple surface movement pattern similar to human TM, and have a maximum displacement limitation at a frequency of $<400 \mathrm{~Hz}$. While the TM composite at $>1000 \mathrm{kHz}$ was reported for organized displacement patterns and a more significant number of maximal displacements. Therefore, the report will definitely improve the understanding of the biomechanical properties of ears, so as to develop better TM grafts and the selection of the ideal biomaterial [35].

Platelet-rich fibrin membrane was used to treat traumatic TM perforations. The healing time, mean air-bone gap, and perforated TM recovery rates using this fibrin membrane were checked and compared with paper patch therapy [36]. The closure of TM perforation in $93 \%$ population was reported in fibrin membrane therapy, while $83 \%$ in the paper patch. Again, total closure was identified in 10 days of implantation in platelet-rich fibrin membrane in $80 \%$ of the population, when compared to paper patch therapy, it was only $53 \%$. The hearing was improved by $14.1 \mathrm{~dB}$ in platelet-rich fibrin membrane therapy and $12.4 \mathrm{~dB}$ in patch therapy. Hence, the platelet-rich fibrin membrane's intervention for TM regeneration as a successful model for the treatment of TM perforations [36].

Poly(glycerol subacetate) (PGS) is also widely used in TM regeneration [37]. The healing effect of PGS against gel-film was reported with greater efficacy (91\%) as compared to that of $75 \%$ in gel-film myringoplasty. Endoscopic images (Figure 3) shows that the perforated TM and the TM after 6 weeks of treatment with PGS. The red circle indicates

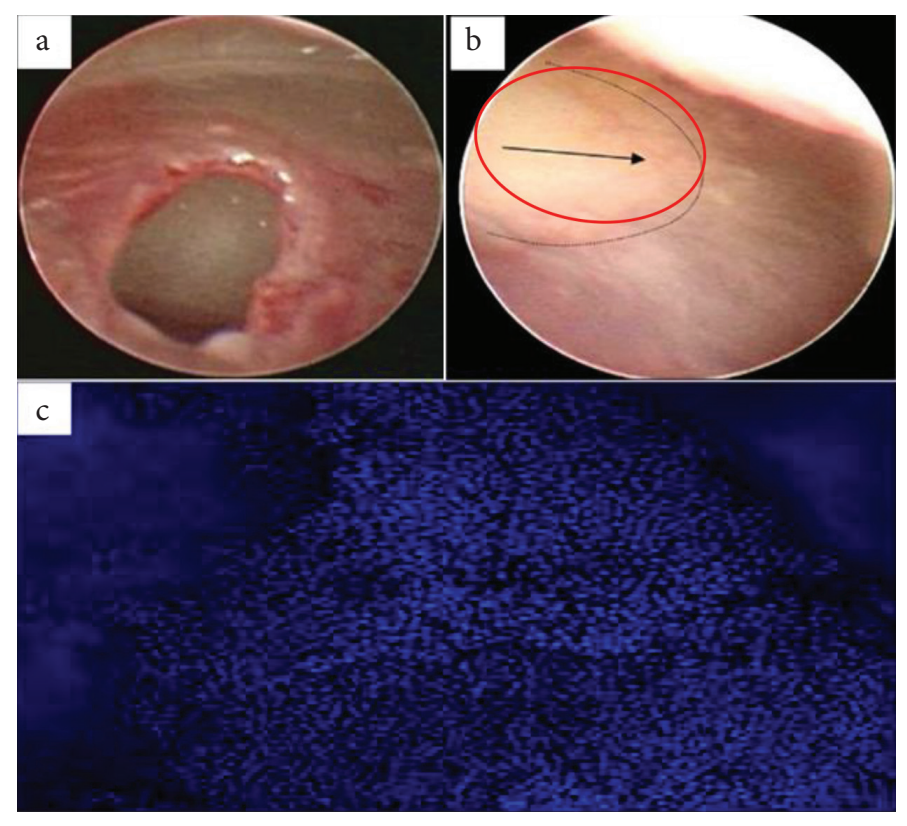

Figure 3 (a) and (b) Endoscopic images of perforated Tympanic Membrane (TM) and healed TM, respectively. (c) Poly(glycerol subacetate) (PGS) plug stained with DAPI indicating the cellular layer over the plug [37].

closure of TM, within 6 weeks duration. A considerable amount of cellularity was expected, and this was confirmed by the staining technique by using 4',6-diamidino-2-phenylindole (DAPI) (Figure 3).

Mota et al. [38] fabricated polymeric biomimetic scaffold using electrospinning and Additive Manufacturing (AM) technologies for 
TM regeneration. PLGA copolymer and polyethylene terephthalate/PBOT/PBT block copolymer were used to manufacture single/ double/tri-scale scaffolds [38]. The combination of AM and electrospinning strategy proved for greater mechanical stability to the scaffold than customized electrospinning technology. Apart from the improved mechanical properties, AM and electrospinning-based scaffolds were reported for better cell adhesion. On the other hand, the triple scale biomimetic scaffold showed good hMSC's disposition, which can be linked to the peculiar arrangement of the collagen fibrils in TM [1,38]. All in all, the finding opened a new dimension in the bio-fabrication of the tissue mimetic scaffold to replace the autologous graft and help in the treatment of the large TM membrane perforations via a tissue engineering approach.

Recently, stem cell-based therapies have found an incredible triumph in the field of tissue regeneration. With the help of two assays, von Unge et al. [39] showed the use of embryonic stem cells in improving the healing of TM perforations. Perforated TM was treated with embryonic stem cells on one ear and with a control substance on the other ear in the first assay. In the second assay, they used the same study but with fluorescent-labelled stem cells. They reported that stem cell treatment encourages complete closure of perforations with considerable mechanical strength. The use of fluorescentlabelled stem cells made it easier to visualize near the area of the perforations, which were again found to be inconsiderate amount [39]. Researchers have used stem cells implanted with various polymers for regenerating TM, and as a result, a considerable amount of success was reported. Different reports on polymers with stem cells suggest that they can effectively modulate stem cell behavior, further regulating tissue growth, cell differentiation, and proliferation. The Transmission Electron Microscopy (TEM) and light microscopic images in Figure 4 are evident in how the thickness of lamina propria has improved by using stem cells $[40,41]$. The highlighted red circles indicated how the thickness had improved when TM was regenerated by using stem cells. Therefore, stem cell-based therapy is a useful alternative for TM regeneration.
In another study PEOT/PBT copolymer based electrospinning scaffold with stem cells (MSCs) was used for TM regeneration [42]. This scaffold has a diameter of $1.9 \pm 0.9 \mu \mathrm{m}$, thickness, and porosity of $220 \pm 56 \mu \mathrm{m}$ and $80 \pm 0.8 \%$, respectively. It was reported that the better adhesion of TM keratinocytes to the electrospun scaffold with greater viability even after 2 days of therapy. This study concluded that using electrospinning, along with bioreactor culture, was an efficient way for eardrum regeneration and re-epithelialization [42].

\subsection{Growth Factor Loaded Biomaterials}

In recent times, growth factors are widely used for effective tissue regeneration. Growth factors are believed to provide the biological basis in the tissue-engineered scaffold. Usually, they are used along with various polymers for achieving their purpose. Seonwoo et al. developed a chitosan patch that releases the EGF. The release of the growth factor results in improved healing when compared to that of spontaneous healing of TM [43]. Another study involving the polymeric composite of the chitosan-polyethyleneimine patch that simultaneously releases EGF and EGF Receptor (EGFR) gene was found [44]. Eventually, the TM cells move more efficiently toward the patches and adhere well to the patches due to the presence of Polyethyleneimine (PEI). It was reported that the chitosan patch, along with PEI, was more hydrophilic and gave a positive charge to the patch compared to that of the chitosan patch alone. This concluded that PEI, EGF, and EGFR gene are responsible for better adhesion of TM cells toward the patch, which improves the healing of TM perforations and ultimately resulted in efficient regeneration of perforated TM (Figure 5) [44].

Further, in the development, differentiation, and the regeneration of various tissue systems, scientists have been giving major emphasis on essential FGF Basic Fibroblast Growth Factor (b-FGF) [11]. The gelatin sponges were reported along with b-FGF for TM regeneration. It was observed that $98.1 \%$ population of the b-FGF group was
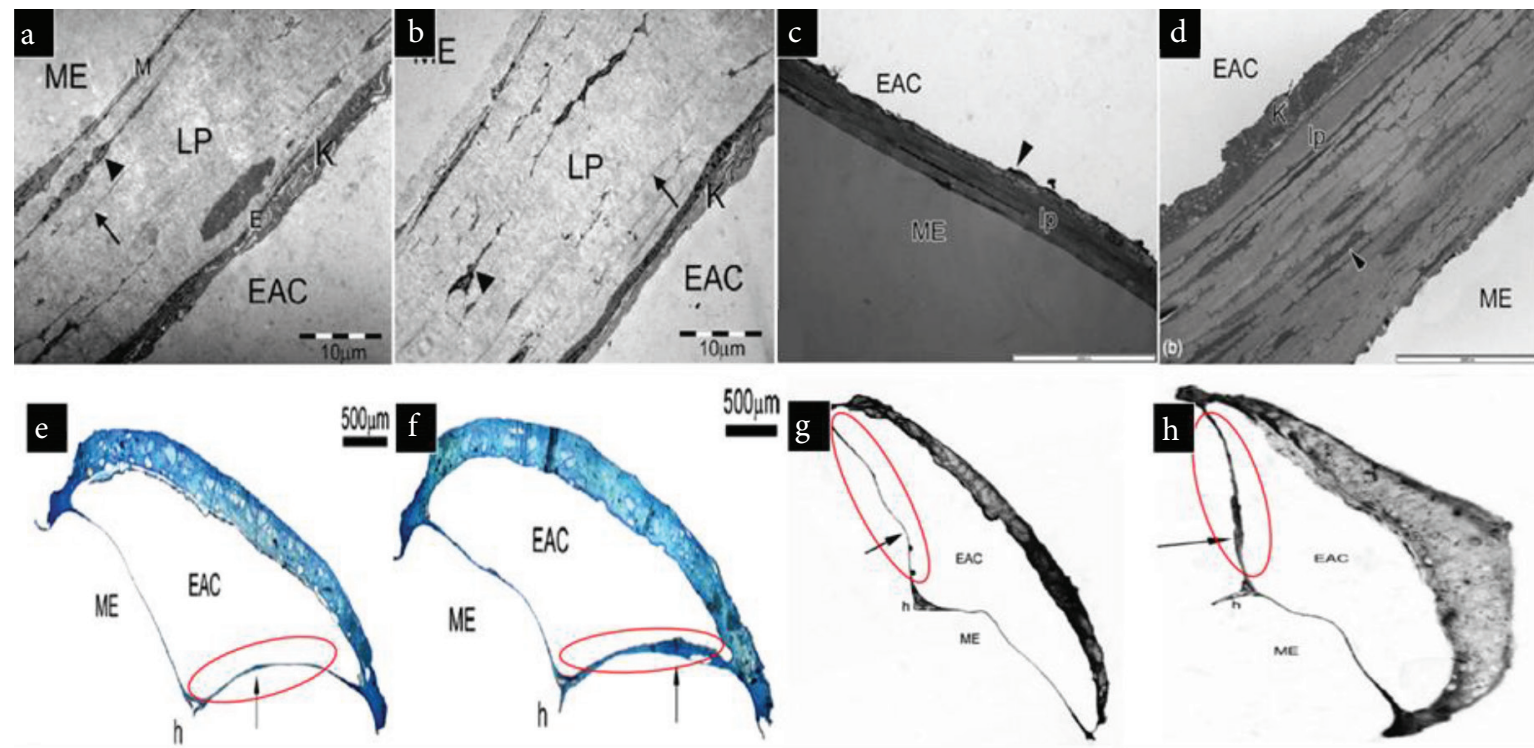

Figure 4 TEM images of (a and c) untreated Tympanic Membrane (TM) (control), (b and d) stem cell treated TM. Light microscopic images of (e and g), Untreated TM ( $\mathrm{f}$ and $\mathrm{h}$ ) stem cell treated TM showing improved thickness and effectiveness in the healing of TM due to stem cell therapy. Red circles indicate TM $[40,41]$. 


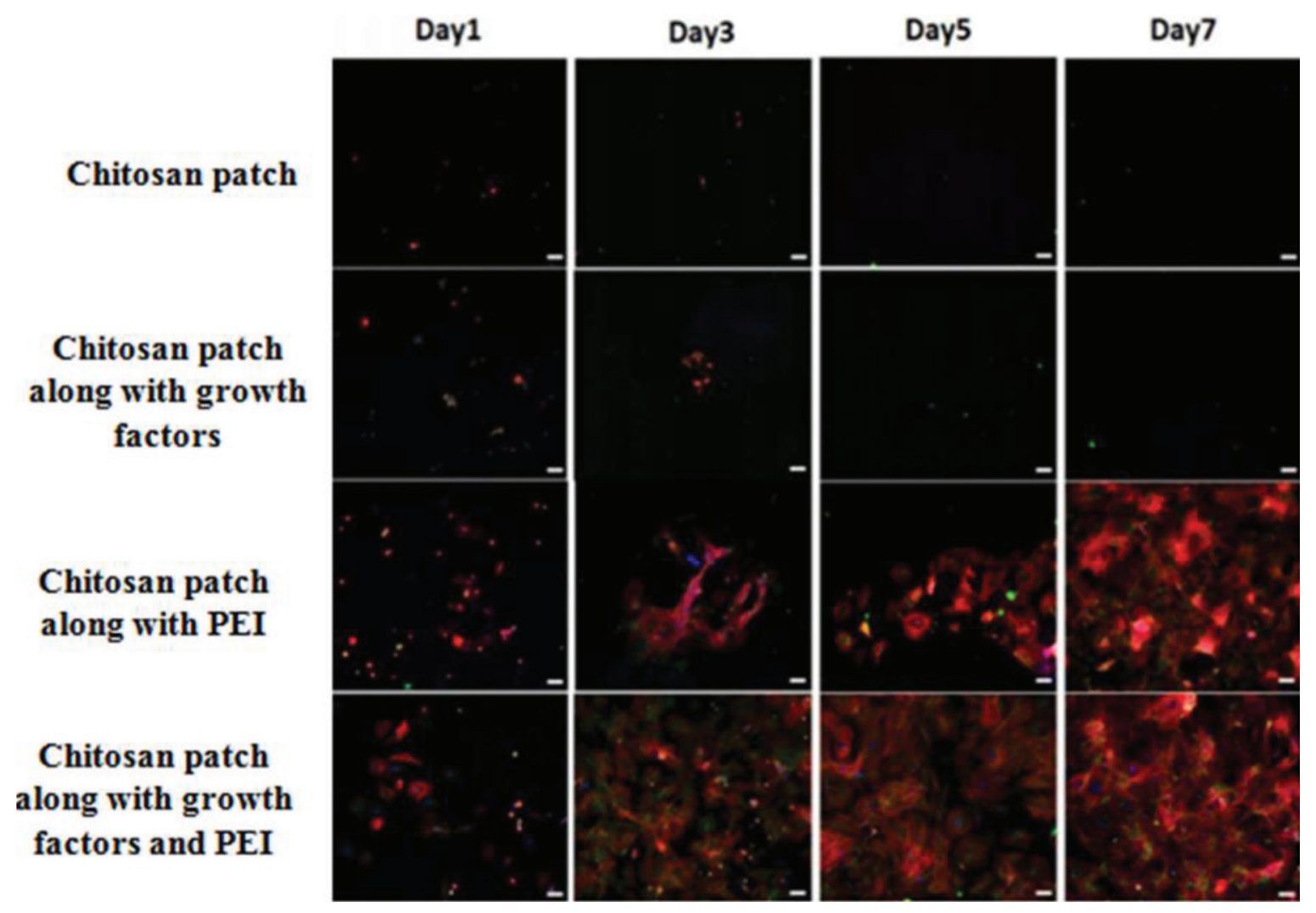

Figure 5 Fluorescence images showing how Polyethyleneimine (PEI) enhances the healing process in Tympanic Membrane (TM) perforations [44].

identified for total closure of TM as compared to that of the control group (only gelatin sponges) which was only $10 \%$ closure. Besides, the hearing levels were improved in patients with the b-FGF treated group. The patients suffering from tinnitus (51/53) and aural fullness (46/53) showed dramatic improvement in their condition after the therapy, $98 \%$ of the population noticed an improvement in tinnitus, and $96.5 \%$ population showed improvement from aural fullness in contrast to that of the control group that showed only $10 \%$ $(1 / 10)$ and $11.1 \%(1 / 9)$ improvement in tinnitus and aural fullness, respectively. Omae et al. [45] looked into the safety, efficacy of the gelatin sponge immersed in this b-FGF for regenerating TM in disease conditions like perforations. They conducted multicentre clinical trials to evaluate safety and efficacy. Results showed no adverse events due to the gelatin sponge immersed in b-FGF. Also, the mean bone conduction threshold improved after the 12 weeks of the regeneration procedure when compared with that of the baseline threshold. From this it can be concluded that not only the gelatin sponge along with b-FGF is safe and efficacious but also is effective in regenerating TM. To concrete, this analogy, another novel biomaterial-driven approach toward TM regeneration, was found, which involves the use of atelocollagen/silicone bilayer membrane as a substrate material (also known as pelnac). Transferrin, a recombinant b-FGF, was incorporated into this pelnac, which efficiently resulted in the regeneration of TM [46]. These results were compared with that of the control group in which b-FGF was replaced with saline. This study's key finding was that the hearing improved by $13.3 \mathrm{~dB}$, TM perforation closed within 3.7 weeks in the b-FGF treated group, and closure was observed in $100 \%$ population. The regeneration was much faster compared to that of gelatin sponge immersed saline. Another study was obtained, which showed the use of vascular endothelial growth factor (VEGF) in TM's regeneration. This study found that VEGF was comparatively better in terms of healing of TM perforations as compared to that of b-FGF [47]. Although, much more research is to be done before completely comparing the two growth factors. The PDGF is well established in tissue regeneration and also needs to be reviewed in TM $[48,49]$. Sang et al. showed the effectiveness of PDGF-AA in regeneration therapy. The thickness of the TM treated with PDGF-AA was observed more than that of the normal TM's thickness. Also, there was an improved healing rate as compared to that of the surgical process [49]. The increase in thickness was explained by the continuous growth of fibroblast in the connective tissue layer, which was found to be maximum at the 9th day of the treatment, resulting in improved mechanical properties. Similarly, results reported by Sang et al. [49] showed that there was no significant difference obtained in healing rate and perforation size when compared with a placebo. Hence, we cannot completely say that using PDGF is beneficial over surgical therapy with such limited studies. Although this further provides scope for researchers in the field of regeneration. Heparinbinding EGF was also used in regenerating TM. Researchers confirmed the effective use of this growth factor in regenerative techniques as the vibration generated from TM after the treatment was similar to that of normal TM [50]. The advantage of this growth factor from others was in terms of efficacy and also in terms of stimulation of the keratinocyte progenitor cells. Another advantage was in terms of mechanism of proliferation as they act through both EGFR dependent and independent mechanisms.

Therefore, the discussion may provide a deep insight into the different hearing conditions related to $\mathrm{TM}$ and biomaterial-driven 
therapies. It also gives an idea of the effect of growth factors in the treatment of TM perforations and other diseases. Hence, with the advancement in material sciences and constant exploration of growth factors use in regenerative medicine may be utilized for TM regeneration. However, by saying this, certain loopholes in the growth factors-loaded therapy like denaturation under extreme treatments, toxicity through rDNA production, high cost of research, and quality control cannot be ignored.

Biological scaffolds were amongst one of the fascinating leads toward the regeneration of TM in conditions like perforations. One such biological scaffold used for the study was the Urinary Bladder Matrix (UBM), which emerged as an effective tool in regenerating TM [51]. In this study, myringoplasty was performed by keeping the left ear as control and the right one receiving the UBM patch. Tissue analysis of TM showed considerable variation in the morphology in terms of uniformity. The treated TM was observed to be more uniform in thickness as compared to that of the control (Figure 6a). These changes in thickness were due to the collagen content in the middle connective tissue layer. The collagen content in the control group was found to be less in the 4th week as compared to that in the 8 th and 12 th week. The result of this was that the TM appeared thicker in the 12th week than that in the 4 th. These observations were when compared to UBM patched TM, the collagen content was more in the patched TM as that in the control group (Figure 6b). A thicker keratinized layer was observed in the UBM patched TM. In terms of cellular growth, the patched TM was found to be effective than that of controlled healing. Hence, it could be said that due to the thickness of TM observed after treatment, keratinized layer over TM, and also cell growth, the UBM scaffold is effective in healing TM perforations (Figure 6c), which ultimately results in optimum vibrations of TM and an effective way of tissue regeneration of TM.

\section{BIOMATERIALS FOR AUDITORY CANAL REGENERATION}

A relay of techniques has been reported for auditory canal regeneration, such as growth factor loaded biomaterial therapy $[52,53]$, novel polymeric biomaterial driver regenerative therapies $[19,26,53]$. The auditory nerve is also crucial for a sense of hearing. Reconstruction of the auditory nerve, by means of inducing neural differentiation and fiber growth in progenitor cells is discussed elsewhere [54]. Here this section will give specific emphasis on the broad therapy discussing above for AC regeneration. To mimic the physiological condition, extensive research is going on the development of polymeric scaffolds. As discussed earlier, the developed scaffolds should exhibit certain characteristics, in vitro like scaffold morphology and porosity, mechanical properties such as Young's modulus, biological characteristics, and in vivo characteristics like the healing effect of scaffold and in vivo animal studies on scaffolds [19]. Here in this section, we discussed the reported materials and their composites for the renewal of the AC.

\subsection{Biomaterials Driven Regeneration}

Currently, the biomaterial-based on the polymer is used for the reconstruction of the EAC wall, but the ear canal still experiences resorption and extrusion. For this reason, a novel technique a

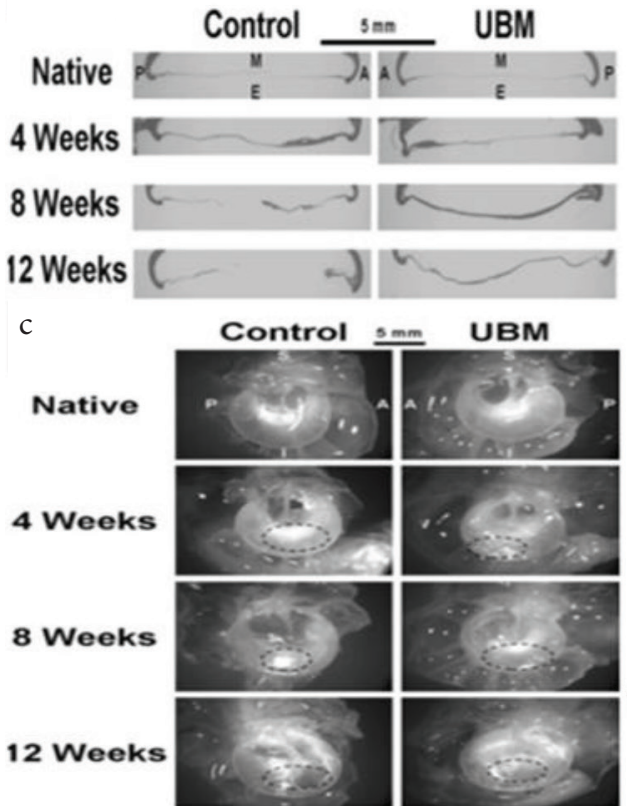

$\mathrm{b}$

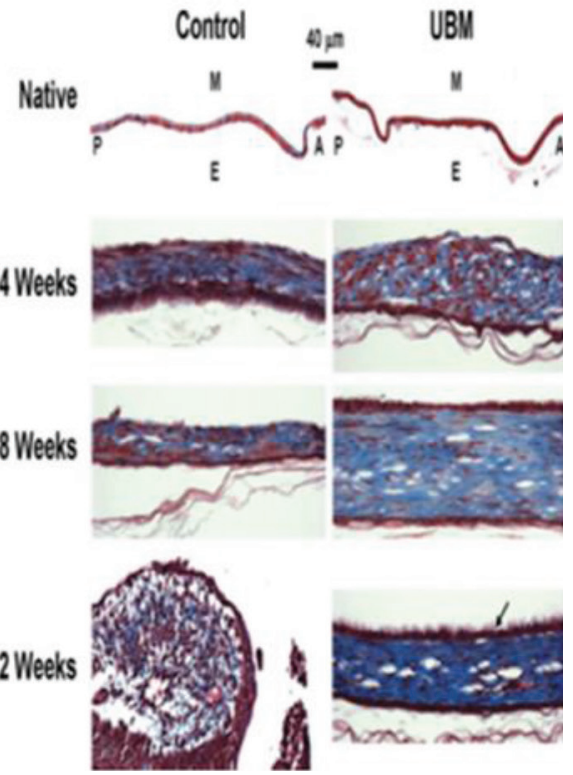

Figure 6 (a) The thickness of Tympanic Membrane (TM) by haematoxylin and eosin stain. (b) Thickness of TM by Masson's trichome staining. (c) Dissecting microscopical images of TM showing uniform thickness of TM, improved healing, and more collagen content of the TM when compared to control [51]. 
was used for the fabrication of polymeric scaffold for EAC reconstruction [19]. PEOT/PBT is reported as a third-generation block copolymer mostly used in regeneration therapy. It was first used to replace eardrum in the rat models [55]. In vivo implantation in rats shows that the copolymer is not toxic, has only a slight foreign body reaction, and can integrate with host tissues. The PEOT/PBT composite reported for the reconstruction of EAC by epithelialization, and it showed 50\% biodegradation within 1 year [55]. The reported research used this biomaterial under middle ear infections caused by Staphylococcus aureus, indicating that it has the potential for otology applications, and verified the application prospects of the PEOT/PBT copolymer family in EAC wall regeneration. Mota et al. [19] designed and fabricated EAC wall by 3-Dimensional Fiber (3DF) an additive manufacturing technique to restore the EAC [19]. The required size and shape of scaffold for a particular patient are imaged by the technique like magnetic resonance imaging and Computer Tomography (CT) [19]. After which the scaffold of the required dimension is designed by Computer Aided Design (CAD) (Figure 7). The first mold of the EAC wall was designed and manufactured by using bulk polymer (Delrin) by using a computer proscribed appliance; after that, fabrication of the porous scaffold of EAC was performed by (3DF) deposition technique by using Bioplotte ${ }^{\mathrm{TN}}$ [19]. The PEOT/PBT scaffold was developed using the 3DF method, with a thickness of $1.72 \pm 0.23 \mathrm{~mm}$, a diameter of $200 \pm 33 \mu \mathrm{m}$, and a weight and volume porosity of $55 \%$ and $39.5 \%$, respectively. The scaffolds have a surface-to-volume ratio of eight times more than that of the bulk device sufficient to interact with the surrounding tissue. In vitro experiments were performed to examine preliminary biological interaction of the scaffold with hMSC's under osteogenic commitment. It was reported that the cells were viable and were producing a thick layer mineral matrix alongside the nodules [19]. Hence, this

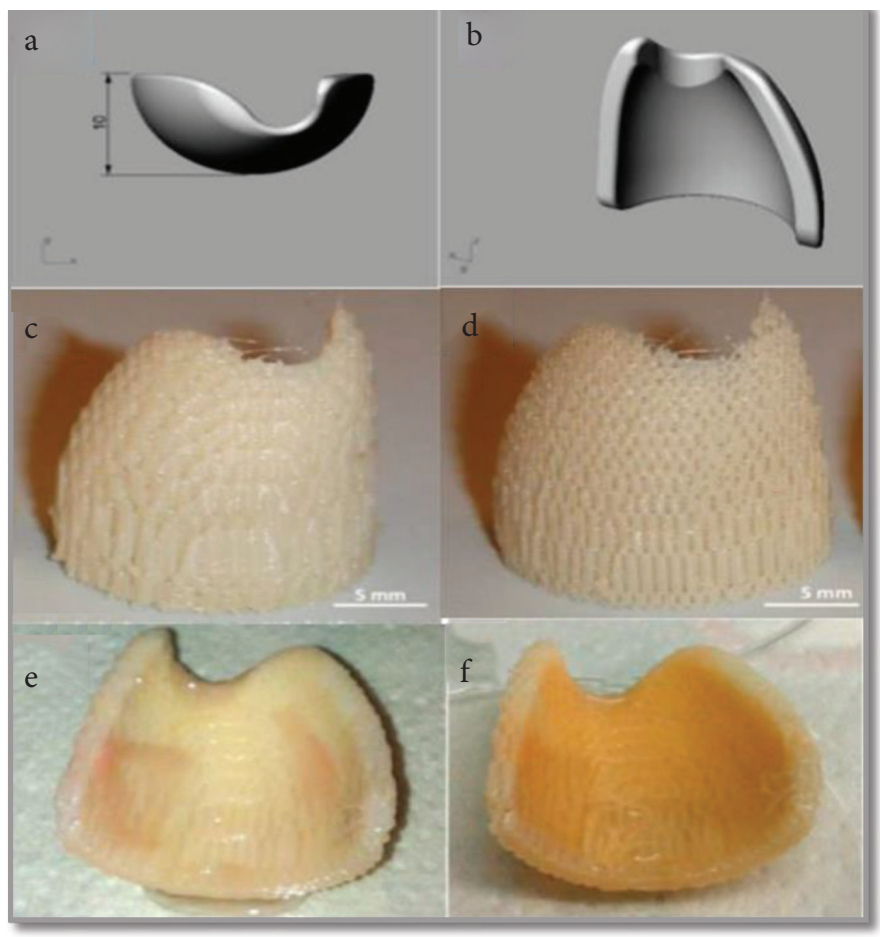

Figure 7 Scaffold architecture (a and b) 3D CAD mold software (SOLID WORKS) (c and d) EAC scaffold produced by 3D method (e) viability staining of the EAC wall scaffold (1st week) (f) viability staining of the EAC wall scaffold (2nd week) [19]. novel 3DF developed scaffold opened a new way for the development of ontological devices using tissue engineering.

Some bioactive materials were also reported as their ability to recover the surgical obliteration. Bio-composite containing Polyglycolic Acid (PGA) sheet using the fibrin glue can facilitate epithelialization after the middle ear surgery in case of Canal Wall Down (CWD) tympanoplasty surgery. This study was mainly done for the treatment of middle ear Cholesteatoma [53]. A thin sheet is made up of poly- $N$-acetyl-glucosamine obtained from snow crabs. They could induce histocyte and fibroblast proliferation, followed by fine collagen production. The sheet was reported to use in covering the reconstructed TM or mastoid bowl surface subsequent to CWD tympanoplasty. But the study reported that epithelialization of the mastoid cavity is deliberate when PGA sheet with fibrin glue is used in CWD tympanoplasty. The benefit of the PGA polymer is biodegradable, which disappear within 100 days when applied. PGA is degraded by means of pyruvate by hydrolysis to water and carbon dioxide in the body. The result of the reconstruction of the canal wall followed by CWD tympanoplasty was reported, which is shown in Figure 8 [53]. The mechanism of regeneration of the ear canal with the PGA sheet was not clearly known in the reported article. They consider that the PGA sheet act as a scaffold that retains fibrin and exudates from the bony surface, the fibroblasts from bony exudates move toward the fibrin matrix and reproduce, which might assist the production of collagen fibers [53]. Conversely, the action of the PGA sheet will diminish within 3 months, as the sheet gets degraded.

The scaffolds have certain desirable properties such as good mechanical strength, biocompatibility, tunable surface chemistry, structural similarity as that of native tissue. In order to achieve these properties, scaffolds can be fabricated by classical solvent casting to the most recent 3D-printing technology. The comparative information of these techniques is summarised in Table 1.
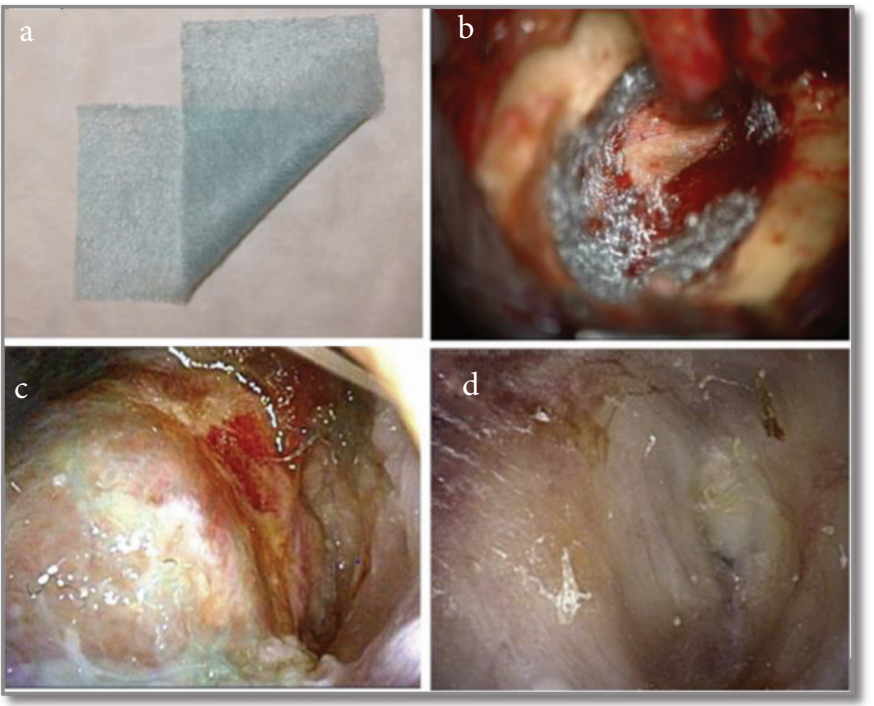

Figure 8 Operational and post-operational findings in treated Canal Wall Down (CWD) tympanoplasty with Polyglycolic Acid (PGA) polymer and fibrin (a) PGA sheet. (b) Piece of PGA sheet attached to the bone surface using fibrin after completion of CWD tympanoplasty. (c) Postoperatively epithelialization observed after 25 days. (d) Epithelialization was observed after 12 weeks postoperatively [53]. 
Table 1 Summary of scaffold fabrication methods

\begin{tabular}{|c|c|c|c|c|c|}
\hline Sr. No. & Method & Characteristic feature & Limitation & Application & References \\
\hline 1 & $\begin{array}{l}\text { Solvent casting/salt } \\
\text { leaching method }\end{array}$ & $\begin{array}{l}\text { Biodegradability and porosity } \\
\text { can be controlled }\end{array}$ & $\begin{array}{l}\text { Low mechanical properties, } \\
\text { residual of solvents, and } \\
\text { porogen material }\end{array}$ & $\begin{array}{l}\text { Bone and cartilage tissue } \\
\text { engineering }\end{array}$ & {$[56]$} \\
\hline 2 & $\begin{array}{l}\text { Template synthesis } \\
\text { method }\end{array}$ & $\begin{array}{l}\text { Use the nonporous membrane } \\
\text { as a template }\end{array}$ & $\begin{array}{l}\text { Unable to form a long and } \\
\text { continuous fibers }\end{array}$ & $\begin{array}{l}\text { Bone tissue engineering, } \\
\text { scaffolding by CAD }\end{array}$ & {$[57]$} \\
\hline 3 & Electrospinning process & $\begin{array}{l}\text { High surface area and drug } \\
\text { carrier }\end{array}$ & $\begin{array}{l}\text { Difficult to make a large } \\
\text { volume of scaffolds and } \\
\text { limited mechanical strength }\end{array}$ & $\begin{array}{l}\text { Synthetic skin and patch for } \\
\text { wound healing }\end{array}$ & [42] \\
\hline 4 & Freeze drying & $\begin{array}{l}\text { 3D porous, durable, flexible } \\
\text { and cost-effective }\end{array}$ & $\begin{array}{l}\text { Uniform porosity is not } \\
\text { maintained, and long } \\
\text { processing time }\end{array}$ & $\begin{array}{l}\text { Bone tissue regeneration, } \\
\text { and complicated scaffold } \\
\text { preparation }\end{array}$ & [29] \\
\hline 5 & $3 \mathrm{D}$ printing & $\begin{array}{l}\text { Complex object prototyping } \\
\text { made easy and offers high } \\
\text { mechanical strength }\end{array}$ & Low geometrical control & Any tissue & [58] \\
\hline
\end{tabular}

\section{TECHNIQUES USED FOR FABRICATION OF SCAFFOLDS}

The scaffolds have certain advantages in regeneration regarding biocompatibility, surface chemistry, tunable mechanical properties, and function. The different methods are employed for scaffold fabrication, and the methods are summarized in Table 1 . These are well-established techniques and nicely reviewed in various literature too.

\section{CONCLUSION}

The TM and AC regeneration methods based on tissue engineering have multiple uses. This article reviews the biomaterial-driven approach, which has heaped up extensive concentration in TM and $\mathrm{AC}$ regeneration. Replacement of the surgical process with the proposition of using biomaterials in regenerative therapy gives the impression of being more pragmatic. Polymers, such as collagen, chitosan, alginate, PGA, PEG, PEOT/PBT, and PCL, etc., can be a good choice. However, to enhance the bioactivity and accelerate the regeneration, these polymers are combined with the growth factors and cells. The ideal tissue-engineered scaffolds should have good biocompatibility, biodegradability, bioactivity, and should mimic the structural and mechanical similarity with the native tissue. To this end, various manufacturing techniques are used, for instance, solvent casting, electrospinning, and 3D printing. However, these biomaterials show an ample amount of good results but more research is needed in the field of TM and AC regenerative tissue engineering. Major advancement is deemed necessary for the development of the smart biomaterial, cell-free approach, and decellularized biomaterial. Combining this biopolymer with smart additive or bioactive material may become the preferred strategy for $\mathrm{TM}$ and $\mathrm{AC}$ regeneration.

\section{FUTURE PROSPECTS}

Until today, both TM and AC have been treated extensively through surgery. Myringoplasty and tympanoplasty are the major surgical procedures widely used for the served purpose. However, poor patient compliance, pain, surgical costs, and postoperative infections severely limit their success. In order to minimize the invasiveness of the treatment of TM and AC, tissue regeneration through biomaterials has significantly conquered a great interest. The combined use of scaffolds, cells and various biochemical factors has undoubtedly successfully replaced flapped out biological tissues and improved people's quality of life. Scaffolding technology can indeed mimic the microenvironment of an organization. Due to the various limitations of natural, synthetic, organic and inorganic materials in tissue engineering, its scope may shift to smart biological materials. Smart biological materials enable materials to adapt to physical, chemical, magnetic and electrical environmental changes. Some materials that may be more interested in $\mathrm{TM}$ and $\mathrm{AC}$ regeneration are Polyvinylidene difluoride (PVDF), Poly(3-hydroxybutyrate-co-3-hydroxyvalerate) (PHBV), poly( $N$-isopropylacrylamide), and barium titanate. Selfhealing polymers from smart materials and various hydrogel-based therapies may also become new methods for TM and AC regeneration. Nanocomposite scaffolds composed of polymers and ceramics will also be explored in this field. From this point of view, a lot of research is still needed to find ideal materials and scaffolding technology for the treatment of ear dysfunction. A lot of research into this field, especially the research of Danti et al., makes it only a matter of time before we minimize the gap in tissue regeneration.

\section{CONFLICTS OF INTEREST}

The authors declare they have no conflicts of interest.

\section{AUTHORS' CONTRIBUTION}

DR, SA and NM collected, analyzed, and drafted the information related to tympanic membrane regeneration and its fabrication techniques. Similarly, RP and VS analyzed and drafted the information related to auditory canal regeneration and related scaffold fabrication techniques. ARH contributed in improvement in the manuscript in terms of conceptualization, and final drafting of the manuscript. GK suggested the topic and further supervision in terms of corrections and finalization of the manuscript. 


\section{REFERENCES}

[1] Danti S, D’Alessandro D, Mota C, Bruschini L, Berrettini S. 18 - Applications of bioresorbable polymers in skin and eardrum. In: Perale G, Hilborn J, editors. Bioresorbable Polymers for Biomedical Applications. Amsterdam, The Netherlands: Woodhead Publishing; 2017, pp. 423-444.

[2] Tortora GJ, Derrickson BH. Principles of anatomy and physiology. United Kingdom: John Wiley \& Sons; 2008.

[3] Kristensen S. Spontaneous healing of traumatic tympanic membrane perforations in man: a century of experience. J Laryngol Otol 1992;106:1037-50.

[4] Spiegel JH, Kessler JL. Tympanic membrane perforation repair with acellular porcine submucosa. Otol Neurotol 2005;26:563-6.

[5] Park MK, Kim KH, Lee JD, Lee BD. Repair of large traumatic tympanic membrane perforation with a Steri-Strips patch. Otolaryngol Head Neck Surg 2011;145:581-5.

[6] Rüedi L. Cholesteatoma formation in the middle ear in animal experiments. Acta Otolaryngol 1959;50:233-42.

[7] Gladstone HB, Jackler RK, Varav K. Tympanic membrane wound healing. An overview. Otolaryngol Clin North Am 1995;28:913-32.

[8] Johnson AP, Smallman LA, Kent SE. The mechanism of healing of tympanic membrane perforations. Acta Otolaryngol 1990;109: 406-15.

[9] Stenfors LE, Carlsöö B, Salén B, Winblad B. Repair of experimental tympanic membrane perforations. Acta Otolaryngol 1980;90:332-41.

[10] Jellinge ME, Kristensen S, Larsen K. Spontaneous closure of traumatic tympanic membrane perforations: observational study. J Laryngol Otol 2015;129:950-4.

[11] Kanemaru S, Umeda H, Kitani Y, Nakamura T, Hirano S, Ito J. Regenerative treatment for tympanic membrane perforation. Otol Neurotol 2011;32:1218-23.

[12] Dabholkar JP, Vora K, Sikdar A. Comparative study of underlay tympanoplasty with temporalis fascia and tragal perichondrium. Indian J Otolaryngol Head Neck Surg 2007;59:116-9.

[13] Glasscock ME 3rd, Kanok MM. Tympanoplasty—a chronological history. Otolaryngol Clin North Am 1977;10:469-77.

[14] Camnitz PS, Bost WS. Traumatic perforations of the tympanic membrane: early closure with paper tape patching. Otolaryngol Head Neck Surg 1985;93:220-3.

[15] Hong P, Bance M, Gratzer PF. Repair of tympanic membrane perforation using novel adjuvant therapies: a contemporary review of experimental and tissue engineering studies. Int J Pediatr Otorhinolaryngol 2013;77:3-12.

[16] Teh BM, Marano RJ, Shen Y, Friedland PL, Dilley RJ, Atlas MD. Tissue engineering of the tympanic membrane. Tissue Eng Part B Rev 2013;19:116-32.

[17] Bagot d’Arc M, Daculsi G, Emam N. Biphasic ceramics and fibrin sealant for bone reconstruction in ear surgery. Ann Otol Rhinol Laryngol 2004;113:711-20.

[18] Dornhoffer J, Simmons O. Canal wall reconstruction with Mimix hydroxyapatite cement: results in an animal model and case study. Laryngoscope 2003;113:2123-8.

[19] Mota C, Milazzo M, Panetta D, Trombi L, Gramigna V, Salvadori $\mathrm{PA}$, et al. 3D fiber deposited polymeric scaffolds for external auditory canal wall. J Mater Sci Mater Med 2018;29:63.

[20] Goncalves S, Bas E, Langston M, Grobman A, Goldstein BJ, Angeli S. Histologic changes of mesenchymal stem cell repair of tympanic membrane perforation. Acta Otolaryngol 2017;137:411-16.
[21] Seonwoo H, Kim SW, Shin B, Jang KJ, Lee M, Choo OS, et al. Latent stem cell-stimulating therapy for regeneration of chronic tympanic membrane perforations using IGFBP2-releasing chitosan patch scaffolds. J Biomater Appl 2019;34:198-207.

[22] Wu S, Peng H, Li X, Streubel PN, Liu Y, Duan B. Effect of scaffold morphology and cell co-culture on tenogenic differentiation of HADMSC on centrifugal melt electrospun poly (L-lactic acid) fibrous meshes. Biofabrication 2017;9:044106.

[23] Salerno A, Di Maio E, Iannace S, Netti PA. Tailoring the pore structure of PCL scaffolds for tissue engineering prepared via gas foaming of multi-phase blends. J Porous Mater 2012;19:181-8.

[24] Sealy C. Scaffold shape gets nerve cells into the groove. Mater Today 2016;19:136-7.

[25] Koppes RA, Park S, Hood T, Jia X, Abdolrahim Poorheravi N, Achyuta $\mathrm{AH}$, et al. Thermally drawn fibers as nerve guidance scaffolds. Biomaterials 2016;81:27-35.

[26] Deschamps AA, Grijpma DW, Feijen J. Poly(ethylene oxide)/ poly(butylene terephthalate) segmented block copolymers: the effect of copolymer composition on physical properties and degradation behavior. Polymer 2001;42:9335-45.

[27] Ratner BD, Hoffman AS, Schoen FJ, Lemons JE. Introduction: Biomaterials Science. In: Hoffman AS, Ratner BD, Schoen FJ, Lemons JE, editors. Biomaterials Science: An Introduction to Materials in Medicine. 3rd ed. Netherlands: Elsevier Inc.; 2013.

[28] Kim JH, Choi SJ, Park JS, Lim KT, Choung PH, Kim SW, et al. Tympanic membrane regeneration using a water-soluble chitosan patch. Tissue Eng Part A 2009;16:225-32.

[29] Kim J, Kim SW, Choi SJ, Lim KT, Lee JB, Seonwoo H, et al. A healing method of tympanic membrane perforations using three-dimensional porous chitosan scaffolds. Tissue Eng Part A 2011;17:2763-72.

[30] Levin B, Redmond SL, Rajkhowa R, Eikelboom RH, Atlas MD, Marano RJ. Utilising silk fibroin membranes as scaffolds for the growth of tympanic membrane keratinocytes, and application to myringoplasty surgery. J Laryngol Otol 2013;127:S13-S20.

[31] Shen Y, Redmond SL, Teh BM, Yan S, Wang Y, Atlas MD, et al. Tympanic membrane repair using silk fibroin and acellular collagen scaffolds. Laryngoscope 2013;123:1976-82.

[32] Shen Y, Redmond SL, Teh BM, Yan S, Wang Y, Zhou L, et al. Scaffolds for tympanic membrane regeneration in rats. Tissue Eng Part A. 2012;19:657-68.

[33] Lee H, Jang CH, Kim GH. A polycaprolactone/silk-fibroin nanofibrous composite combined with human umbilical cord serum for subacute tympanic membrane perforation; an in vitro and in vivo study. J Mater Chem B 2014;2:2703-13.

[34] Moscato S, Rocca A, D’Alessandro D, Puppi D, Gramigna V, Milazzo M, et al. Tympanic membrane collagen expression by dynamically cultured human mesenchymal stromal cell/starbranched poly ( $\varepsilon$-caprolactone) nonwoven constructs. Appl Sci 2020;10: 3043 .

[35] Kozin ED, Black NL, Cheng JT, Cotler MJ, McKenna MJ, Lee DJ, et al. Design, fabrication, and in vitro testing of novel threedimensionally printed tympanic membrane grafts. Hear Res 2016;340:191-203.

[36] Gür ÖE, Ensari N, Öztürk MT, Boztepe OF, Gün T, Selçuk ÖT, et al. Use of a platelet-rich fibrin membrane to repair traumatic tympanic membrane perforations: a comparative study. Acta Otolaryngol 2016;136:1017-23.

[37] Wieland AM, Sundback CA, Hart A, Kulig K, Masiakos PT, Hartnick CJ. Poly (glycerol sebacate)-engineered plugs to repair 
chronic tympanic membrane perforations in a chinchilla model. Otolaryngol Head Neck Surg 2010;143:127-33.

[38] Mota C, Danti S, D’Alessandro D, Trombi L, Ricci C, Puppi D, et al. Multiscale fabrication of biomimetic scaffolds for tympanic membrane tissue engineering. Biofabrication 2015;7:025005.

[39] von Unge M, Dirckx JJ, Olivius NP. Embryonic stem cells enhance the healing of tympanic membrane perforations. Int J Pediatr Otorhinolaryngol 2003; 67:215-9.

[40] Rahman A, Olivius P, Dirckx J, Von Unge M, Hultcrantz M. Stem cells and enhanced healing of chronic tympanic membrane perforation. Acta Otolaryngol 2008;128:352-9.

[41] Rahman A, von Unge M, Olivius P, Dirckx J, Hultcrantz M. Healing time, long-term result and effects of stem cell treatment in acute tympanic membrane perforation. Int J Pediatr Otorhinolaryngol 2007;71:1129-37.

[42] Danti S, Mota C, D’alessandro D, Trombi L, Ricci C, Redmond $\mathrm{SL}$, et al. Tissue engineering of the tympanic membrane using electrospun PEOT/PBT copolymer scaffolds: a morphological in vitro study. Hear Balance Commun 2015;13:133-47.

[43] Seonwoo H, Kim SW, Kim J, Chunjie T, Lim KT, Kim YJ, et al. Regeneration of chronic tympanic membrane perforation using an EGF-releasing chitosan patch. Tissue Eng Part A 2013;19: 2097-107.

[44] Lee MC, Seonwoo H, Garg P, Jang KJ, Pandey S, Park SB, et al. Chitosan/PEI patch releasing EGF and the EGFR gene for the regeneration of the tympanic membrane after perforation. Biomater Sci 2018;6:364-71.

[45] Omae K, Kanemaru SI, Nakatani E, Kaneda H, Nishimura T, Tona $\mathrm{R}$, et al. Regenerative treatment for tympanic membrane perforation using gelatin sponge with basic fibroblast growth factor. Auris Nasus Larynx 2017;44:664-71.

[46] Hakuba N, Taniguchi M, Shimizu Y, Sugimoto A, Shinomori Y, Gyo K. A new method for closing tympanic membrane perforations using basic fibroblast growth factor. Laryngoscope 2003;113:1352-5.

[47] Cho KS, Lee DG, Shin DH, Park YD, Chon KM. The importance of vascular endothelial growth factor in the healing of acute tympanic membrane perforation. Am J Otolaryngol 2010;31:309-14.
[48] Röösli C, von Büren T, Gassmann NB, Huber AM. The impact of platelet-derived growth factor on closure of chronic tympanic membrane perforations: a randomized, double-blind, placebocontrolled study. Otol Neurotol 2011;32:1224-9.

[49] Yeo SW, Kim SW, Suh BD, Cho SH. Effects of platelet-derived growth factor-AA on the healing process of tympanic membrane perforation. Am J Otolaryngol 2000;21:153-60.

[50] Santa Maria PL, Kim S, Varsak YK, Yang YP. Heparin bindingepidermal growth factor-like growth factor for the regeneration of chronic tympanic membrane perforations in mice. Tissue Eng Part A 2015;21:1483-94.

[51] Parekh A, Mantle B, Banks J, Swarts JD, Badylak SF, Dohar JE, et al. Repair of the tympanic membrane with urinary bladder matrix. Laryngoscope 2009;119:1206-13.

[52] Kanemaru Si. Regeneration of the soft tissue defects of the external auditory meatus. In: Ito J, editor. Regenerative Medicine in Otolaryngology. Japan, Tokyo: Springer; 2015, pp. 33-46.

[53] Kobayashi T, Gyo K, Komori M, Hyodo M. Polyglycolic acid sheet attached with fibrin glue can facilitate faster epithelialization of the mastoid cavity after canal wall-down tympanoplasty. Auris Nasus Larynx 2017;44:685-9.

[54] Rask-Andersen H, Boström M, Gerdin B, Kinnefors A, Nyberg G, Engstrand T, et al. Regeneration of human auditory nerve. In vitro/in video demonstration of neural progenitor cells in adult human and guinea pig spiral ganglion. Hear Res 2005;203:180-91.

[55] Bakker D, van Blitterswijk CA, Hesseling SC, Daems WT, Kuijpers W, Grote JJ. The behavior of alloplastic tympanic membranes in Staphylococcus aureus-induced middle ear infection. I. Quantitative biocompatibility evaluation. J Biomed Mater Res 1990;24:669-88.

[56] Villar-Fernandez MA, Lopez-Escamez JA. Outlook for tissue engineering of the tympanic membrane. Audiol Res 2015;5:117.

[57] Laidlaw DW, Costantino PD, Govindaraj S, Hiltzik DH, Catalano PJ. Tympanic membrane repair with a dermal allograft. Laryngoscope 2001;111:702-7.

[58] Kuo CY, Wilson E, Fuson A, Gandhi N, Monfaredi R, Jenkins A, et al. Repair of tympanic membrane perforations with customized bioprinted ear grafts using chinchilla models. Tissue Eng Part A 2018;24:527-35. 